\title{
Evaluación técnica de la agregación de valor a partir de variedades de cebolla (Allium cepa) cultivadas al norte de la Región Central Oriental de Costa Rica
}

\section{Technic evaluation of value-adding processes of onion (Allium cepa) materials grown in the north part of the Central-Oriental Region of Costa Rica}

\section{Laura Patricia Brenes-Peralta ${ }^{1}$, Marianella Gamboa-Murillo², Ana Cecilia Segreda-Rodríguez ${ }^{3}$}

Fecha de recepción: 26 de setiembre de 2018

Fecha de aceptación: 3 de noviembre de 2018

Brenes-Peralta, L; Gamboa-Murillo, M; Segreda-Rodríguez, A. Evaluación técnica de la agregación de valor a partir de variedades de cebolla (Allium cepa) cultivadas al norte de la Región Central Oriental de Costa Rica. Tecnología en Marcha. Vol. 32-3. Julio-Setiembre 2019. Pág. 126-134.

DOI: https://doi.org/10.18845/tm.v32i3.4503

1 Máster en Gerencia y Gestión Ambiental e Ingeniera Agropecuaria Administradora con énfasis en empresas agropecuarias. Escuela de Agronegocios, Instituto Tecnológico de Costa Rica. Costa Rica. Correo electrónico: labrenes@tec.ac.cr. (D) https://orcid.org/0000-0002-5169-9961

2 Máster en Educación Técnica e Ingeniera Agropecuaria Administradora con énfasis en empresas agroindustriales. Escuela de Agronegocios, Instituto Tecnológico de Costa Rica. Costa Rica. Correo electrónico magamboa@tec.ac.cr. (iD) https://orcid.org/0000-0002-3831-8730

3 Máster en Gerencia de Programas Sanitarios en Inocuidad de Alimentos, Instituto Nacional de Innovación y Transferencia en Tecnología Agropecuaria, Convenio INTA-Instituto Tecnológico de Costa Rica. Costa Rica. Correo electrónico: asegreda@inta.go.cr. 


\title{
Palabras clave
}

Agregación de valor; agroindustrialización; alimento; mínimamente procesado; procesamiento.

\section{Resumen}

El sector cebollero costarricense es dinámico y cuenta con una institucionalidad que respalda las acciones de investigación y extensión, como el Programa Nacional de Cebolla del Ministerio de Agricultura y Ganadería-MAG, las Agencias de Extensión Agropecuaria locales, y el Programa de Investigación y Transferencia de Tecnología Agropecuaria-PITTA Cebolla. Sin embargo, la comercialización en fresco de la cebolla enfrenta dificultades, por lo que este proyecto tuvo por objetivo "Evaluar el proceso de agregación de valor a variedades de cebolla (Allium cepa) cultivadas en zona Norte de la Región Central Oriental (al norte de Cartago). Se consideraron los materiales E515, Predator y Alvara, valorando dos variables principales para el análisis comparativo a partir de pruebas de valor agregado, considerando: tiempo de ejecución del proceso agroindustrial y rendimiento de producción. Las variedades Predator y E515 usualmente mostraron los rendimientos más altos y los menores tiempos de elaboración según el producto de valor agregado del que se tratara. Los resultados fueron transferidos a los beneficiarios en un taller teórico y tres sesiones prácticas en la Planta Piloto Agroindustrial del Instituto Tecnológico de Costa Rica (TEC). Se recomienda ahondar en investigaciones para validar los resultados hallados y se sugieren estudios de factibilidad específicos en el caso de desear incursionar en emprendimientos de este tipo.

\section{Keywords}

Value adding; agroindustry; food; minimal procesing; food processing.

\begin{abstract}
The Costa Rican onion sector is a dynamic one, supported by an institutional framework dedicated to research and extension, such as the National Onion Programme from the Agriculture and Livestock Ministry-MAG, the Local Extension Agencies and the Research and Technology Transference Programme (PITTA-cebolla). The fresh onion commercialization faces challenges; therefore, this project proposed to evaluate the value adding process for three onion (Allium cepa) materials, cultivated in the northern zone of the Central-Oriental Region of Costa Rica. E515, Predator y Alvara materials were assessed and compared under two main variables for value adding product development: processing yield and time. The Predator and E515 onions showed the higher yields and lesser processing times in comparison to the evaluated materials. Results were transferred to beneficiaries within the focus region through a workshop including a theory session and three practices at the Agro-Industry Pilot Plant at TEC. Further research is suggested to validate this first outcomes, and specific feasibility studies are encouraged for future entrepreneurship initiatives.
\end{abstract}

\section{Introducción}

Los Agronegocios contemplan las diferentes etapas de la agrocadena que van de la finca a la mesa, pasando por producción, agregación de valor, distribución, y consumo, asociados a su vez por servicios, estructuras y elementos que respaldan la toma de decisiones. Pasar del concepto tradicional de agricultura, sin considerar procesos de gestión que planifiquen, organicen, ejecuten, direccionen, y controlen y evalúen las actividades en que se incursione, 
supone riesgos para el crecimiento y permanencia de esas actividades. Por esto, identificar información útil en las fases de planeamiento y pre-inversión [1] son claves para el desarrollo de proyectos y emprendimientos, como podrían ser aquellos de agroindustrialización y de agregación de valor.

Existen varios mecanismos para agregar valor a la producción primaria, como la transformación o la diferenciación, entre otros [2]. Por ello, tanto las que tienden a una mínima transformación o al procesamiento agroindustrial de la cebolla son de interés para la correspondiente agrocadena costarricense. Lo anterior se fundamenta en la posibilidad de lograr mejores condiciones de comercialización, negociación, ingreso y vida útil al producto agrícola. En 2012, el Programa Agroambiental Mesoamericano impulsado por el Centro Agronómico Tropical de Investigación y Enseñanza (CATIE) en países de la región tales como Guatemala, señalaba en sus hallazgos la importancia del empoderamiento de la población rural para lograr mediante el conocimiento, la innovación y la agregación de valor, una dinamización de las agrocadenas y por tanto el desarrollo rural [3].

La zona Norte de la Región Central Oriental costarricense, integrada mayor mente por porciones de los cantones de Alvarado y Oreamuno de la provincia costarricense de Cartago, está catalogada como un área rural y agrícola. Existen antecedentes que sitúan a Tierra Blanca (una localidad del cantón de Oreamuno) como un área cuyo manejo del sistema productivo se considera superior al de otras zonas agrícolas (al menos en la cuenca del Río Reventado), lo que relaciona esta condición con un mejor nivel organizacional de los productores y la posibilidad de agroindustrialización [4]. Por lo anterior, parte de los grupos beneficiarios así como la selección de los materiales de este proyecto, provinieron de esa zona, al tiempo que se trata de un área relevante en la producción de cebolla a nivel nacional [5]. Si bien existe una robusta institucionalidad y algunas agremiaciones de productores identificadas para el sector cebollero nacional, la comercialización en fresco enfrenta dificultades típicas relacionadas a la oferta, demanda y perecibilidad del producto [5].

Costa Rica registra una producción anual de cebolla relativamente constante, con una leve tendencia al alza en los últimos años. La cebolla generó cerca del 0,4 por ciento en el Valor Agregado Agropecuario, con un consumo per cápita de 8 toneladas, generando así empleos directos e indirectos [5]. Se ha observado que precio al productor, en la feria y al mayorista tiende a decrecer a lo largo de los años, mientras que el precio al consumidor ha mostrado una tendencia al alza o incremento. La balanza comercial para este caso específico, es negativa en lo referente al hecho de que la búsqueda de ventajas competitivas, la innovación y la agregación de valor pueden cobrar importancia. Según datos de SEPSA y el Consejo Nacional de Producción (CNP), el sector cebollero está integrado por 730 productores en la Región Central Oriental o lo que es lo mismo, un 84\% de los productores nacionales [5].

A lo largo de la última década se ha desarrollado investigación en esta agrocadena, usualmente bajo enfoques agronómicos y de control biológico [6] [7] [8]. Sin embargo, a nivel de estudios propiamente direccionados a la agregación de valor, las publicaciones científicas para el caso costarricense no son abundantes, localizando estudios documentados como el proyecto denominado "Desarrollo de un producto de cebolla mínimamente procesado" [9]. El mismo formó parte de la iniciativa sectorial "Cultivares de cebolla con mayor potencial productivo y de rentabilidad para la región Central" ejecutado mediante el Convenio INTA-ITCR, el cual Ilegó a determinar que existía potencial técnico para el desarrollo de cebolla mínimamente procesada [9] con las variedades Gladalan brown y E-515".

A raíz de lo anterior, esta investigación, ejecutada por proyectistas de la Escuela de Agronegocios del Tecnológico de Costa Rica (TEC) en convenio con el Instituto de Innovación y Transferencia de Tecnología Agropecuaria (INTA), con el financiamiento de la Fundación para el Fomento de 
la Investigación y la Transferencia de Tecnología Agropecuaria (FITTACORI), tuvo por objetivo evaluar técnica y económicamente el proceso de agregación de valor a tres variedades de cebolla (Allium cepa) cultivadas en la Región Central Oriental (al norte de Cartago), incluyendo un componente de transferencia de tecnología a beneficiarios de la zona. Esto último respondió igualmente a uno de los fines de las instancias participantes en el proyecto, como fueron los grupos beneficiarios de Corporación Hortícola Nacional (CHN), CoopeBaires, la Asociación de Horticultores del Irazú (ASHORI), y entidades de apoyo al proyecto como el Programa Nacional de Cebolla del Ministerio de Agricultura y Ganadería (MAG), las Agencias de Extensión Agropecuaria de Tierra Blanca y Pacayas, y el Programa de Investigación y Transferencia de Tecnología Agropecuaria-(PITTA) Cebolla.

\section{Metodología}

El proyecto se llevó a cabo durante el año 2017, principalmente en la Planta Piloto Agroindustrial (PPA) de la Escuela de Agronegocios del TEC, ubicada en el Cantón Central de la Provincia de Cartago, en el distrito Oriental, Barrio Los Ángeles 951'16'N 8354'33'O. Además, se coordinó con extensionistas de las Agencias de Extensión de la Zona Norte de Cartago (Región Central Oriental), principalmente de Tierra Blanca para obtener muestras de campo de las variedades de cebolla.

Inicialmente, se realizaron observaciones de campo, lluvias de ideas, consideraciones según criterio de experto y revisiones de literatura para seleccionar potenciales productos con valor agregado a base de cebolla. Adicionalmente, se realizaron pruebas preliminares de los diferentes productos posibles para darle valor agregado a la cebolla, los cuales se llevaron a una sesión del PITTA Cebolla para su validación. Paralelamente, se realizó un sondeo con actores clave (gerencia del Programa Nacional, PITTA Cebolla y productores) para visualizar posibles mejoras de los productores desarrollados y seleccionar la materia prima (materiales de cebolla con los que se trabajaría), resultando estos en cebolla registrada en la Oficina Nacional de Semilla (ONS) como E-515, Alvara y Predator. También se entrevistó a estos actores relevantes para conocer el manejo dado a la materia prima.

Una vez cosechada y secada la cebolla de manera tradicional por los productores que aportaron muestras al proyecto, se caracterizó la materia prima mediante análisis proximales, y cualitativos, cotejados estos últimos contra la descripción otorgada por el reglamento "RTCR 69:2.000. Cebolla seca" [10]. En cuanto al análisis proximal se evaluaron las variables de la humedad $(\%)$, ceniza $(\% \mathrm{~m} / \mathrm{m})$, proteína $(\% \mathrm{~m} / \mathrm{m})$, fibra total $(\% \mathrm{~m} / \mathrm{m})$, grasa total $(\% \mathrm{~m} / \mathrm{m})$ y carbohidratos $(\% \mathrm{~m} / \mathrm{m})$, tomando de referencia los métodos analíticos del Asociación Oficial de Químicos Analíticos (A.O.A.C por sus siglas en inglés), 16va Edición de 1995.

Dicha materia prima se sometió a pruebas de procesamiento agroindustrial en la PPA del TEC, a saber: cebolla mínimamente procesada empacada al vacío, cebolla caramelizada en aceite con romero, y aderezo con cebolla y vegetales. Se realizaron al menos repeticiones de cada proceso para obtener una formulación, ajustando cada vez elementos según variables sensoriales observadas, criterio de experto y consulta a terceros (panel no entrenado sin una formalización de análisis sensorial) para llegar a la formulación final. Una vez definida esta, se inició con el proceso de estandarización de cada producto, lo cual consistió de procesamientos por triplicado para cada producto con cada variedad. Los tratamientos se codificaron como P1, P2 y P3 para las tres repeticiones con cebolla Predator, A1, A2, A3 para las repeticiones de la cebolla Alvara y E1, E2, E3 para las repeticiones de la cebolla E-515. Durante la realización de dichas repeticiones, se tomaron datos para respaldar variables tales como $\mathrm{pH}$, sólidos solubles totales ( ${ }^{\circ}$ Brix), rendimiento (\%), costo ( $\left.\phi / g r a m o\right)$, tiempo (min) y equipo utilizado. A nivel del 
análisis comparativo, se seleccionó puntualmente observar las variables de rendimiento y tiempo de proceso.

En lo referente al análisis sensorial de los productos agroindustriales desarrollados, se evaluó el color, olor, sabor, y apariencia general de éstos, utilizando una escala de 0 a 6 , en donde 0 indicaba que el producto no gustaba del todo y 6 que le gustaba mucho.

También se realizó un análisis del costo productivo en función de productos similares detectados en el mercado nacional. Para la estimación de este parámetro se consideró a) el precio de mercado de la materia prima e insumos, b) el precio de mercado de empaques sugeridos por criterio de experto y basados en la observación de productos similares en mercado, c) el precio de la electricidad (cálculo en función del consumo energético del equipo utilizado y según el proveedor de servicio local) y del agua (cálculo en función del consumo de agua durante el proceso y según el proveedor de servicio local) y e) valor monetario de la mano de obra (en función a la duración del proceso según cada operación y considerado para un colaborador no calificado según el Ministerio de Trabajo en Costa Rica para el 2do semestre 2017). Los costos fueron calculados utilizando hojas de Excel a partir de las variables de precio mencionadas, y su utilización promedio según las formulaciones y diagramas de proceso desarrollados durante las pruebas en la PPA. Se calcularon partiendo de un ejercicio escalado a tandas de $10 \mathrm{~kg}$ de producto final para el producto mínimamente procesado y $6 \mathrm{~kg}$ de producto final para el aderezo de cebolla con vegetales y para la cebolla caramelizada en aceite con romero. Luego se realizó el cálculo en función de presentaciones potenciales del producto según criterio de experto y observación del mercado. Debe recalcarse que se trata de un ejercicio de cálculo de costo operativo, donde no se incluyen otros gastos administrativos, costo de venta y proyección financiera incorporando costos de inversión y financieros. Por esto, en el caso de incursionar en posibles emprendimientos, se recomienda entablar los estudios de factibilidad correspondientes.

Finalmente, se llevaron a cabo actividades de transferencia de tecnología: una teórica y tres prácticas, al subdividir al grupo que asistió a la actividad teórica, con la finalidad de ofrecer una atención más personalizada y mayor interacción entre participantes y extensionistas, al igual que lo hicieron los estudiantes del TEC que participaron en el proceso.

\section{Resultados y discusión}

Se determinó mediante entrevistas con los actores clave (productores, técnicos del MAG) que normalmente el manejo dado a la cebolla consistió de un siembras a campo abierto y de manejo convencional, para determinar posteriormente según el indicador de "días después de siembra" y observación del cultivo el momento de la cosecha. Se determinó, que una práctica agronómica convencional sería el uso de agroquímicos para evitar enfermedades según los periodos de retiro establecidos. Posteriormente, se arrancó la cebolla del suelo sin retirarla del campo para dejar un proceso de secado en el sitio y luego trasladarla a secado en carpas (estructuras usualmente tubulares cubiertas con plástico que permiten aumento de temperatura y aireación para facilitar el secado de las capas externas del bulbo, acondicionándolo para su manejo en adelante dentro de la cadena de valor; igualmente se puede realizar en bodegas). Entre las etapas mencionadas anteriormente, se pueden dar operaciones intermedias de pesaje para determinar rendimiento, selección y clasificación, remoción de hojas conocido como "descolado" y según el mercado de destino se empacará en sacos, mallas, o cajas pudiendo trenzarse o no, siendo esta una práctica cada vez menos frecuente. Como principales cuidados, se mencionó el proceso de secado para evitar ataques de hongos y bacterias que potencialmente generarían pérdida de la cebolla, así como el cuidado por daño mecánico sobre todo en momentos de recolección y pesaje que también afecten el producto. Usualmente, las 
aplicaciones de fungicidas y otros insumos se le realizan a la cebolla de época lluviosa, y debido al alto contenido de humedad que va a tener ésta. El paso de secado no debe ser omitido, el cual usualmente durará al menos 10 días, para asegurar una mayor vida útil del producto.

Posterior al proceso de recibo de la cebolla en la PPA, se procedió a su almacenamiento en estibas de cajas con $15 \mathrm{~kg}$ de producto cada una aproximadamente, en un lugar fresco y sin incidencia de luz directa. Producto de la caracterización de la materia prima, se observan los cuadros 1 y 2 , donde se observan distintas características cualitativas y proximales.

Cuadro 1. Caracterización cualitativa de los materiales de cebolla fresca evaluados

\begin{tabular}{|c|c|c|c|}
\hline Parámetro & Predator & Alvara & E515 \\
\hline Características físicas & $\begin{array}{l}\text { Bulbo redondeado, color } \\
\text { blancuzco amarillento. } \\
\text { Sabor ligeramente dulce. } \\
\text { Se recibe la cebolla seca, } \\
\text { descolada, de tamaño } \\
\text { mediano según la norma } \\
\text { vigente en Costa Rica y } \\
\text { se clasifica como segunda } \\
\text { según las tolerancias de } \\
\text { calidad expresadas en la } \\
\text { norma. }\end{array}$ & $\begin{array}{l}\text { Bulbo redondeado, } \\
\text { color blancuzco. Se } \\
\text { recibe descolada, } \\
\text { siendo cebolla seca, } \\
\text { sabor "más intenso" que } \\
\text { las otras variedades } \\
\text { en estudio, mediana } \\
\text { según la norma } \\
\text { vigente en Costa Rica } \\
\text { y clasificación como } \\
\text { segunda según las } \\
\text { tolerancias de calidad } \\
\text { expresadas en la } \\
\text { norma. }\end{array}$ & $\begin{array}{l}\text { Bulbo redondeado, } \\
\text { color blancuzco } \\
\text { principalmente. Se } \\
\text { recibe descolada, } \\
\text { siendo cebolla seca, } \\
\text { mediana según la norma } \\
\text { vigente en Costa Rica } \\
\text { y clasificación como } \\
\text { segunda según las } \\
\text { tolerancias de calidad } \\
\text { expresadas en la norma. }\end{array}$ \\
\hline
\end{tabular}

Fuente: ONS, 2017 y observaciones durante ejecución del proyecto.

Cuadro 2. Caracterización proximal de los materiales de cebolla fresca evaluados, por cada $100 \mathrm{~g}$ de producto.

\begin{tabular}{|c|c|c|c|}
\hline Parámetro & Predator & Alvara & E515 \\
\hline Humedad \% & 92,4 & $92,6 \%$ & $93,36 \%$ \\
\hline Ceniza $\% \mathrm{~m} / \mathrm{m}$ & 0,4 & 0,4 & 0,3 \\
\hline Proteína $\% \mathrm{~m} / \mathrm{m}$ & 0,6 & 0,6 & 0,4 \\
\hline Fibra total $\% \mathrm{~m} / \mathrm{m}$ & 0,2 & 1,9 & 0,3 \\
\hline Grasa Total \%m/m & 1,1 & 0,5 & 1,1 \\
\hline Carbohidratos $\% \mathrm{~m} / \mathrm{m}$ & 5,5 & 5,9 & 4,9 \\
\hline
\end{tabular}

Fuente: Análisis de proximales ejecutados por Laboratorio Lambda, 2017.

Luego se iniciaron las pruebas preliminares, validación con actores clave y ajuste de formulación. Además, a partir del análisis sensorial aplicado, se determinó que en principio todas las variedades y productos propuestos contaban con una posible aceptación de parte de los consumidores, debiendo explorar posteriormente a profundidad este aspecto.

Particularmente, en el caso de la cebolla mínimamente procesada, se presenta el cuadro 3 respecto al rendimiento y tiempo de procesamiento de cada una de las variedades evaluadas. 
Cuadro 3. Promedio de rendimiento y tiempo de elaboración de Cebolla Mínimamente procesada en la PPA-TEC.

\begin{tabular}{|c|c|c|c|c|c|c|}
\hline & \multicolumn{2}{|c|}{ Predator } & \multicolumn{2}{c|}{ Alvara } & \multicolumn{2}{c|}{ E515 } \\
\hline & rendimiento (\%) & $\begin{array}{c}\text { duración } \\
\text { (min) }\end{array}$ & rendimiento (\%) & $\begin{array}{c}\text { duración } \\
\text { (min) }\end{array}$ & rendimiento (\%) & $\begin{array}{c}\text { duración } \\
\text { (min) }\end{array}$ \\
\hline repetición 1 & $77 \%$ & 78 & $72 \%$ & 70 & $79 \%$ & 64 \\
\hline repetición 2 & $76 \%$ & 84 & $79 \%$ & 74 & $76 \%$ & 78 \\
\hline repetición 3 & $72 \%$ & 64 & $80 \%$ & 70 & $84 \%$ & 67 \\
\hline Media & $75 \%$ & 75 & $77 \%$ & 71 & $80 \%$ & 70 \\
\hline
\end{tabular}

Se observa en la tabla anterior, que la variedad E-515 resultó con un mayor rendimiento de producción, seguida por la cebolla Alvara y por último la Predator. En cuanto al tiempo de elaboración del proceso, el ejecutado con la cebolla E-515 fue el que duró menos, seguido por la cebolla Alvara y finalmente la Predator. Lo anterior se puede deber, según observaciones cualitativas de las proyectistas y asistentes, a una menor dificultad de desprendimiento de catáfilas en la variedad E-515.

En cuanto al segundo producto elaborado, se presenta el cuadro 4.

Cuadro 4. Promedio de rendimiento y tiempo de elaboración de Cebolla Caramelizada en la PPA-TEC

\begin{tabular}{|c|c|c|c|c|c|c|}
\hline & \multicolumn{2}{|c|}{ Predator } & \multicolumn{2}{c|}{ Alvara } & \multicolumn{2}{c|}{ E515 } \\
\hline rendimiento (\%) & $\begin{array}{c}\text { duración } \\
(\mathrm{min})\end{array}$ & rendimiento (\%) & $\begin{array}{c}\text { duración } \\
\text { (min) }\end{array}$ & rendimiento (\%) & $\begin{array}{c}\text { duración } \\
\text { (min) }\end{array}$ \\
\hline repetición 1 & $79 \%$ & 65 & $78 \%$ & 79 & $74 \%$ & 76 \\
\hline repetición 2 & $78 \%$ & 68 & $75 \%$ & 69 & $76 \%$ & 72 \\
\hline repetición 3 & $77 \%$ & 73 & $77 \%$ & 74 & $73 \%$ & 75 \\
\hline Media & $78 \%$ & 69 & $77 \%$ & 74 & $74 \%$ & 74 \\
\hline
\end{tabular}

Se observa que para este caso, el mayor rendimiento con el menor tiempo de elaboración lo generó la cebolla Predator, seguida por la Alvara y finalmente por la E-515. Si bien no se establece un estudio correlacional en este proyecto, podría inferirse alguna relación respecto a los porcentajes de humedad en cada variedad y por tanto la masa final de producto dentro del proceso, así como el tiempo de cocción donde existe evaporación para lograr características deseadas en el producto. Lo anterior debiera ser verificado luego en otros estudios de mayor alcance.

Finalmente, se presenta el cuadro 5 respecto al aderezo de cebolla.

El mayor rendimiento, al igual que para los productos Mínimamente procesados, la variedad E515 mostró el rendimiento más alto. Al igual que en el caso anterior podrían hacerse inferencias sobre los porcentajes de humedad en cada material fresco y la masa final de producto dentro del proceso; sin embargo, en este caso, existe una sumatoria de más insumos frescos que pudieron generar también efectos sobre el rendimiento final. 
Cuadro 5. Promedio de rendimiento y tiempo de elaboración del Aderezo de cebolla en la PPA-TEC

\begin{tabular}{|c|c|c|c|c|c|c|}
\hline & \multicolumn{2}{|c|}{ Predator } & \multicolumn{2}{c|}{ Alvara } & \multicolumn{2}{c|}{ E515 } \\
\hline & rendimiento (\%) & $\begin{array}{c}\text { duración } \\
(\mathrm{min})\end{array}$ & rendimiento (\%) & $\begin{array}{c}\text { duración } \\
(\mathrm{min})\end{array}$ & rendimiento (\%) & $\begin{array}{c}\text { duración } \\
\text { (min) }\end{array}$ \\
\hline repetición 1 & $72 \%$ & 102 & $76 \%$ & 105 & $76 \%$ & 100 \\
\hline repetición 2 & $75 \%$ & 98 & $76 \%$ & 110 & $78 \%$ & 104 \\
\hline repetición 3 & $78 \%$ & 97 & $78 \%$ & 107 & $79 \%$ & 107 \\
\hline media & $75 \%$ & 99 & $77 \%$ & 107 & $78 \%$ & 104 \\
\hline
\end{tabular}

El mayor rendimiento, al igual que para los productos Mínimamente procesados, la variedad E515 mostró el rendimiento más alto. Al igual que en el caso anterior podrían hacerse inferencias sobre los porcentajes de humedad en cada material fresco y la masa final de producto dentro del proceso; sin embargo, en este caso, existe una sumatoria de más insumos frescos que pudieron generar también efectos sobre el rendimiento final.

Respecto al costo productivo, se presenta en el cuadro 6 el costo operativo promedio por tipo de producto desarrollado (no por variedad).

Cuadro 6. Costo promedio por gramo de cada producto con valor agregado según estimaciones del proyecto

\begin{tabular}{|c|c|}
\hline Producto & costo $/ \mathrm{gramo}(\mathbb{K} / \mathrm{g})$ \\
\hline Cebolla mínimamente procesada & 1,36 \\
\hline Cebolla caramelizada en aceite con romero & 3,12 \\
\hline Aderezo de cebolla con vegetales & 3,24 \\
\hline
\end{tabular}

Se detecta que en todos los casos este costo es inferior al valor de mercado de productos similares o de categorías similares en el mercado nacional. Esto dejaría suponer, preliminarmente, que se mantiene un margen potencial para poder considerar otros costos administrativos y de ventas, así como utilidades, debiendo ser cuidadosamente calculados según la operación de la que se tratara.

\section{Conclusiones}

Los resultados del proyecto sugieren que es posible incursionar en actividades de agregación de valor para materiales de cebolla como el E515, Predator y Alvara encontrando distintos rendimientos, tiempos y preferencias de consumidores para cada caso; donde la cebolla Predator probó tener tiempos menores de elaboración para los casos de la cebolla caramelizada en aceite con romero y el aderezo de cebolla con vegetales. La variedad E515 arrojó el menor tiempo de elaboración en mínimamente procesado. La variedad E515 reportó el rendimiento más alto en cebolla mínimamente procesada y aderezo de cebolla con vegetales; para la cebolla caramelizada en aceite con romero se obtuvo mayor rendimiento con la variedad Predator.

El proyecto permitió desarrollar formulaciones requeridas para la evaluación buscada, pero se recomienda que, en caso de incursionar en una actividad comercial, cada grupo empresarial 
o productor individual deberá depurar su idea de negocio, incluyendo la formulación que se ajuste mejor al nicho de mercado al que apunte, el análisis de costo correspondiente y la validación de resultados como los obtenidos en este primero proyecto.

Se rescata así mismo, la importancia del trabajo inter-institucional y la motivación a los grupos productores con información científica y respaldada guíe los procesos en que estos incursionen para agregación de valor, dado el reto de inversión, capacitación y conocimiento que esto implica.

\section{Agradecimientos}

Las proyectistas desean agradecer el apoyo de estudiantes asistentes del TEC Alonso López Serrano, Alonso Navarro González y Andrey Ureña Jiménez, al Máster Carlos Gómez Córdoba de la Planta piloto agroindustrial del TEC, y a los especialistas del MAG Iván Serrano Bulakar (Gerente del Programa Nacional de Cebolla), Jairo González (Agencia de Extensión de Tierra Blanca) y a los productore que aportaron producto para las pruebas y visitas de campo.

\section{Referencias}

[1] J. F. Esquembre y J. Morales, "Como gestionar con éxito su proyecto de certificación profesional”, 2013.

[2] L. P. Brenes-Peralta, "Marco orientador para la agregación de tomate costarricense", Cartago, Costa Rica : Tecnológico de Costa Rica, 2016.

[3] F. Ramírez Agüero, I. A. Gutiérrez-Montes, L. Hernández Hernández, A. Escobedo y D. Padilla, "El empoderamiento de las mujeres en las cadenas de valor: Un reto para las políticas de desarrollo rural", 2012. [En línea]. Available: http://repositorio.bibliotecaorton.catie.ac.cr/bitstream/handle/11554/7979/El_empoderamiento_de_las_mujeres.pdf?sequence=9\&isAllowed=y.

[4] L. Ramírez, A. McHugh y A. Alvarado, "Evolución histórica y caracterización socioeconóimca de la cuenta media edl Río Reventado, Cartago, Costa Rica. Agronomía Costarricense Vol.32, Núm 2, pp 53-72, / 2008

[5] M. I. Morales-Chacón y M. Flores-Fioravanti, "Ficha Técnica Cebolla (Allium cepa)", SEPSA, San José, Costa Rica , 2015.

[6] A. V. Guillén-Watson, R. Chacón-Cerda y C. Zúñiga-Vega, "Detección del virus del enanismo amarillo de la cebolla (OYDV) y el virus latente común del ajo (GCLV) en ajo (Allium sativum L) costarricense", Tecnología en Marcha Vol. 24, Núm. 4 , pp. 47-58, 2011

[7] W. Rivera Méndez y K. Meneses Montero, "Ensayan técnicas de combate sostenibles para controlar pudrición blanca de ajo y cebolla", InvestigaTEC Núm. 23 , pp. 22-23, $2015 .$.

[8] J. Brenes-Madríz y A. Guillén-Watson, "Establecimiento de un protocolo in vitro para el cultivo del ajo (Allium sativum) en Costa Rica" Tecnología en Marcha Vol. 27, N. ${ }^{\circ}$ 4, pp. 40-57, 2014.

[9] A. C. Segreda-Rodríguez y S. Gómez, "Desarrollo de un producto de cebolla mínimamente procesado", Convenio INTA-TEC, Cartago, Costa Rica, 2009.

[10] MEIC y MAG. Reglamento Técnico RTCR 69:2.000. Cebolla Seca. 2003 [En línea]. Available: http://www.mag. go.cr/legislacion/2003/de-31255.pdf 